\title{
Erwachsenenbildung in Österreich
}

\author{
Klaus Heuer
}

Online publiziert: 4. März 2015

(C) Die Autor(en) 2015. Dieser Artikel ist auf Springerlink.com mit Open Access verfügbar.

\section{Rezension zu:}

Werner Filla (2014). Von der freien zur integrierten Erwachsenenbildung. Zugänge zur Geschichte der Erwachsenenbildung in Österreich. Peter Lang Edition, Frankfurt a. M., 271 Seiten, 51,85€, ISBN 978-3-631-63473-8

In seiner Monografie führt Werner Filla in die moderne Geschichte der Erwachsenenbildung in Österreich seit 1870 ein und bestärkt das Erreichte. Er gibt viele Hinweise auf die einschlägige Sekundärliteratur, ist systematisierend, bezogen auf die Richtungen und Träger der Erwachsenenbildung, und praxisbezogen, indem er die Handlungsfelder - und möglichkeiten der Akteure innerhalb der jeweiligen politischen Rahmenbedingungen benennt. Seine Arbeit führt jeweils epochen- und themenbezogen Forschungsdesiderate für eine historische Erforschung der Erwachsenenbildung an. Damit leistet sie eine das Spektrum der Geschichte der modernen Erwachsenenbildung strukturierende und orientierende Pionierarbeit. Darüber hinaus arbeitet Filla treffsicher historisch längerfristige, aber immer noch wirksame Kontinuitätslinien heraus. Diese kenntnisreiche Arbeit stellt eine Fortsetzung und in gewisser Weise auch einen Abschluss der bislang vorliegenden Publikationen des Autors dar, der seine Erfahrungen als ehemaliger Generalsekretär der österreichischen Volkshochschule und als ausgewiesener Publizist der Erwachsenenbildungsgeschichte hier zusammenführt.

Im Rahmen eines gesellschaftstheoretischen Erklärungsansatzes führt Filla aus, dass die historische Entwicklung der Erwachsenenbildung an drei Bedingungen geknüpft ist: an den gesellschaftlichen Reformbedarf, gelungene Institutionalisie-

\footnotetext{
Dr. K. Heuer $(\square)$

Deutsches Institut für Erwachsenenbildung - Leibniz-Zentrum für Lebenslanges Lernen e.V. (DIE)

Bonn, Deutschland

E-Mail: heuer@die-bonn.de
} 
rungsansätze der Reformbestrebungen und an Pioniere, die sich in besonders engagierter und erfolgreicher Weise für die Durchsetzung der Reformen einsetzen (S. 28). Damit liefert er einen Erklärungsansatz, der so klar bislang in keiner Geschichte der Erwachsenenbildung vorgestellt wurde.

Die Einführung ist auch für Lesende aus Deutschland interessant, weil sie die historischen Parallelentwicklungen in Österreich und Deutschland, aber auch Unterschiede und Missverständnisse - wie die fehlende Abgrenzung des in Deutschland geprägten Begriffs der „Arbeitsgemeinschaft“ vom österreichischen Spezifikum der „Fachgruppen“ (S. 68 ff.) - kenntnisreich ausführt. Sie ist jedoch nicht kultur- und bildungsgeschichtlich ausgerichtet und bindet die Erwachsenenbildung nicht in die allgemeine Sozialgeschichte ein. Sie beschränkt sich auf die sogenannte moderne institutionalisierte Erwachsenenbildung und berücksichtigt zum Beispiel nicht die reichhaltigen Erträge der Forschung zur Wissensexpansion in der Neuzeit (R. van Dülmen) und zur Volksaufklärung seit Mitte des 18. Jahrhunderts (H. Böning). Auch fehlt ein internationaler Vergleich und eine Problemorientierung. Insbesondere englische (T. Steele, S. Marriott) und auch kanadische (M.R. Welton) theoriegeleitete Ansätze mit ihren engen Bezügen zum Öffentlichkeitsbegriff von J. Habermas, der von vorpolitischen öffentlichen Lern-Räumen in demokratisierender Absicht spricht - werden nicht berücksichtigt.

Der von Filla verwendete Begriff ,,von unten“ (S. 23, 25, 65, 93, 118) als Lernund Demokratisierungspotential in der Geschichte der Erwachsenenbildung bleibt unscharf - ist weder identifiziert als soziale Bewegung noch als Arbeiteremanzipation oder als Teilnehmermacht. Die Sympathien des Autors gelten der Geschichte der Arbeiterbildung. So urteilt er, dass sowohl die marxistisch orientierte Theorieentwicklung der Erwachsenenbildung als auch ihr emanzipativer Handlungsanspruch und die Demokratisierungsimpulse historisch prägend gewesen seien (S. 21, 82 ff., 108 ff.). Hingewiesen wird hier insbesondere auf die Kritik am Neutralitätsgebot (S. 109) in der Wissensvermittlung.

Wenn man so will, drückt sich schon im Buchtitel die Rahmengeschichte des historischen Entwicklungsmodells der Erwachsenenbildung aus, das Filla in seinem Zugang vertritt. Danach entwickelt sich die Erwachsenenbildung zugespitzt aus „,frei“ im Sinne von ,von unten“ hin zu einer ,integrierten“ Erwachsenenbildung, die im Wesentlichen mittelstandsorientiert und staatlich reglementiert ist. Ihr emanzipativer Kern bleibt auf der Strecke des historisch betrachtet erfolgreichen Durchsetzungsprozesses von Erwachsenenbildung. So gesehen beschreibt die Rahmengeschichte Fillas realistisch und pessimistisch den aktuellen Zustand der Erwachsenenbildung und hält gleichzeitig mit seinem historischen Zugang den Blick für eine demokratisierende Alternative offen. Legt diese Sichtweise Wert auf die Polarität von frei und integrativ, so könnte eine alternative Erkenntnishaltung darin bestehen, das Spannungsverhältnis von frei und integrativ, als Matrix, als Strukturschema zur Einordnung der Geschichte der Erwachsenenbildung ins Spiel zu bringen.

Fillas Einführung ist in den Fragen und Kritikpunkten, die sie evoziert, anregend und weiterführend und auch deshalb allen an der Geschichte der Erwachsenenbildung Interessierten sehr zu empfehlen. 
Open Access Dieser Artikel unterliegt den Bedingungen der Creative Commons Attribution License. Dadurch sind die Nutzung, Verteilung, und Reproduktion erlaubt, sofern der/die Originalautor/en und die Quelle angegeben sind. 